\title{
A Practical Authentication with Key Agreement Protocol for Satellite Communications on ECC
}

\author{
H.H. Huang, J.H. Chen, B.J. Huang \\ School of Mathematics and Statistics, Wuhan University, Wuhan, China
}

\begin{abstract}
In order to remove the pitfalls and enhance the subsequent schemes, a practical authentication scheme is introduced by using elliptic curve cryptosystem (ECC) and identity-based cryptography (IBC) with three-round handshake identification system. It supports seven essential advantages and adequate security attributes. The high performance and countering the strong DoS attack are two main superiorities.
\end{abstract}

KEYWORD: satellite communications; authentication; ECC; DoS attack

\section{INSTRUCTIONS}

Due to geographic and environment limitations, traditional personal communication systems broadcast week and delay signals [1-2]. In the last decade, considerable attention has been paid to the low-earth-orbit satellite communications system (LSC system) for its numbers of advantages: Short transmission delay, small attenuation signal, large communication area and far broadcasting range [29]. The main components in a LSC system are the low-earth-orbit satellites, the network control center (NCC), the gateways and the mobile devices [8]. Recently, we analyze the security aspects of Chang et al's scheme [9] and identify many loopholes that are common of other related scheme [7-12]. Therefore, an enhanced authentication protocol is proposed using elliptic curve cryptosystem (ECC) and identity-based cryptography (IBC) in threeround handshake identification system. The scheme not only provides seven essential requirements but also counter the strong new DoS attack.

The paper is organized as follows. Section 2 gives preliminaries. The scheme is introduced in Section 3. Section 4 discusses the security of the scheme. We draw some concluding remarks in Section 5.

\section{PRELIMINARIES}

\subsection{Elliptic curve cryptosystem (ECC)}

In this paper, we just give a simple description of the ECC defined over a prime field $F_{P}$. A non-singular elliptic curve $F_{P}(a, b)$ over $F_{P}$ is defined by an equation $y^{2}=x^{3}+a x+b(\bmod p) \quad, \quad a, b \in F_{P}$ with the discriminant is $\Delta=4 a^{3}+27 b^{2} \neq 0(\bmod p)$. Then the set $G_{P}=\left\{(x, y) \mid x, y \in F_{P}\right.$ and $\left.(x, y) \in E_{P}(a, b)\right\} \cup\{O\}$ can form a cyclic additive elliptic curve group, where the point $O$ is identity element of $G_{P}$. Let $P$ is a base of $G_{P}$ with an order $n$ as $n P=o$ for the smallest integer $n>0$. The scalar multiplication on the group $G_{P}$ defined as $k P=P+P+\cdots+P(k$ times $)$. Details of elliptic curve group properties are given in [15].

\subsection{Hard computational problem}

- Elliptic curve discrete logarithm problem (ECDLP): Assumed two points $Q, P \in G_{P}$, to find $k \in[1, n-1]$ such that $Q=k P$ is impossible.

- Computational Diffie-Hellman problem (CDHP) Assumed there are three points $P, a P, b P$ for $a, b \in[1, n-1]$, which guarantee the points in $G_{P}$, the computation of $a b P$ is hard to $G_{P}$.

\section{THE PTOPOSED PROTOCOL}

The protocol consists of four phases. The notations used in the paper are defined as follows:

- $U$ : a mobile user $\quad-I D_{U}: U$ 's unique identity

- $p w$ : U's password $\quad x$ : the private key of $N C C$

- ID leos $:$ LEOS's identity $\quad \cdot h(\cdot)$ : one-way hash function

- $S K$ : share session key $\quad N C C$ : a network control center

$\bullet$ : exclusive-or operation $\bullet$ LEOS : a low-earth-orbit satellite 


\subsection{Initialization phase}

The $N C C$ executes this phase as follows:

1) Choose a secure elliptic curve equation $E_{P}(a, b)$ and a generator point $P$ of a cyclic additive elliptic curve group $G_{P}$ with order $n$, where $p$ is a $k$-bit prime number; Select a random number $x \in[1, n-1]$ as its private key and computes the corresponding public key $P_{N}=x P$.

2)Pick a one-way key derivation function $k d f:\{0,1\}^{*} \times\{0,1\}^{*} \times G_{P} \rightarrow\{0,1\}^{k}$ and define an operation $a \circ A=\left(a \oplus x_{A}, y_{A}\right), a \in Z_{P}^{*}, A=\left(x_{A}, y_{A}\right) \in G_{P}$. Publish $\left\{E_{P}(a, b), n, P, P_{N}, h(\cdot), k d f\right\}$ as the system parameters and keeps its private key $x$ secret.

\subsection{Registration phase}

If a mobile user $U$ wants to register at the system, this phase is performed only once as follows:

1) $U$ freely chooses his valid identity $I D_{U}$ and password $p w$ to compute $y=h\left(I D_{U}, p w\right)$. Then he sends the message $\left\{I D_{U}, y\right\}$ to NCC through a secure channel.

2) When receives the message, $N C C$ selects a nonce $N_{0}$ as the initial temporary identity for $U$. Then $N C C$ computes $S=\left(x+h\left(I D_{U}\right)\right)^{-1} P, \quad L=y \circ S$ and $v=h\left(y, I D_{U}\right)$. Finally, NCC stores $\left\{N_{0}, I D_{U}\right\}$ into the verifier-table and delivers the message $M_{0}=\left\{N_{0}, L, v\right\}$ to $U$ via a secure channel.

\subsection{Authentication phase}

When $U$ intends to login the system, he inputs his $I D_{U}$ and $p w$ into his device. Then, this phase is performed as follows:

1) The device computes $y=h\left(I D_{U}, p w\right)$ and compares whether $h\left(y, I D_{U}\right)=? v$ or not. If they are unequal which means $U$ inputs incorrect $I D_{U}$ or $p w$, it stops and prompts user input again; or else, it selects a variable $r_{1} \in[1, n-1]$ to compute $R=r_{1} P, Q=r_{1}(y \circ L)$ and $\alpha=h\left(N_{0}, I D_{U}, R, Q\right)$. Then, the device sends the request message $\left\{N_{0}, R, \alpha\right\}$ to $L E O S$.

2) Upon receiving the message, $L E O S$ shifts it and its identity $I D_{\text {leos }}$ to $N C C$.

3) On receiving $\left\{N_{0}, R, \alpha, I D_{\text {leos }}\right\}, N C C$ uses $N_{0}$ to find the matching $I D_{U}$ in the verifier-table. If cannot find, $N C C$ refuses the request; or else, it computes $Q^{\prime}=\left(x+h\left(I D_{U}\right)\right)^{-1} R, \quad \alpha^{\prime}=h\left(N_{0}, I D_{U}, R, Q^{\prime}\right)$ and checks the condition $\alpha^{\prime}=? \alpha$. If the condition fails, $N C C$ refuses this session; otherwise, it generates a nonce $N_{1}$ and computes the session key $S K=k d f\left(N_{0}, I D_{U}, Q^{\prime}\right), \quad \beta=h\left(N_{1}, S K\right)$. Then $N C C$ sends $\left\{N_{1}, \beta, I D_{\text {leos }}\right\}$ to $L E O S$.

4) LEOS shifts the message $\left\{N_{1}, \beta\right\}$ to $U$. when $U$ receives the response message, he computes $S K^{\prime}=k d f\left(N_{0}, I D_{U}, Q\right), \quad \beta^{\prime}=h\left(N_{1}, S K^{\prime}\right)$ and checks the condition $\beta^{\prime}=$ ? $\beta$. If it fails, $U$ refuses $N C C$; otherwise, $U$ stores $N_{1}$ next to $N_{0}$ in the device. Finally, $U$ computes $\delta=h\left(N_{0}, N_{1}, I D_{U}, S K^{\prime}\right)$ and sends $\{\delta\}$ to LEOS.

5) LEOS shifts $\left\{\delta, I D_{\text {leos }}\right\}$ to NCC. When NCC gets the message, it computes $\delta=h\left(N_{0}, N_{1}, I D_{U}, S K\right)$ and checks the condition $\delta=? \delta$. If the condition holds, it means $U$ has received the next temporary identity $N_{1}$ and shares the session key $S K$. Hence, $N C C$ can update $N_{0}$ as $N_{1}$ and apply $S K$ to encrypt the exchange data with $U$. Otherwise, this session is terminated.

$U$ stores $N_{1}$ next to $N_{0}$ rather than updates $N_{0}$ as $N_{1}$ in step 4. It means $U$ will keep $N_{0}$ until receive his next temporary identity $N_{2}$. Then, $U$ updates $N_{0}$ as $N_{2}$. As Fig. 2, this technique is used to overcome the 'De-synchronization challenge'.

\subsection{Password change phase}

This phase is activated whenever $U$ needs to replace his password. Firstly, $U$ inputs $I D_{U}$ and $p w$ to the device and asks for changing the password. Then the device will automatically perform as follows:

1) The device computes $y=h\left(I D_{U}, p w\right)$ and compares whether $h\left(y, I D_{U}\right)=? v$ or not. If they are not equal, it refuses the request. Otherwise, $U$ is asked to input a new password $p w_{\text {new }}$.

2) The device computes $y_{\text {new }}=h\left(I D_{U}, p w_{\text {new }}\right)$, $L_{\text {new }}=y_{\text {new }} \circ(y \circ L)$ and $v_{\text {new }}=h\left(y_{\text {new }}, I D_{U}\right)$. Finally, it replaces the original $v, L$ using $v_{\text {new }}, L_{\text {new }}$ separately, which completes this phase.

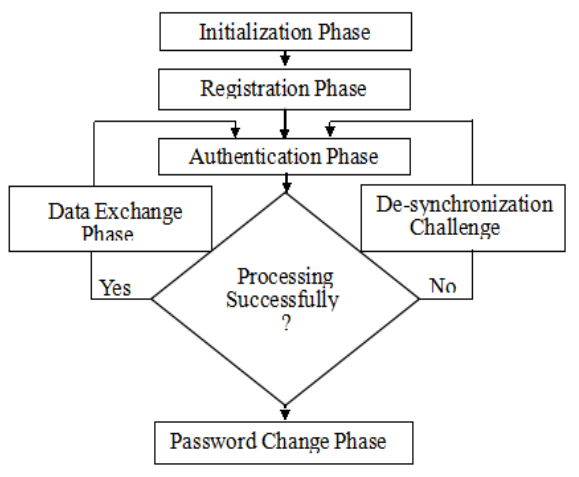

Fig.1 Flow diagram of the protocol 


\section{SECURITY ANALYSIS OF THE PROTOCOL}

\subsection{Valid mutual Authentication}

Through the first and third handshake, NCC authenticates $U$ because just the legal $U$ who holds a real identity $I D_{U}$ and corresponding password $p w$ can compute valid $\alpha$ and $\delta$ for verification; through the second handshake, $U$ authenticates $N C C$ because only the true $N C C$ can generate $\beta$ with the master key $x$ and $U$ 's identity $I D_{U}$. Meanwhile, the messages $M_{1}, M_{2}$ and $M_{3}$ are authenticated to come from the original sender. For example, the values $N_{0}$ and $R$ in $M_{1}$ are sure to come from $U$ since they are bound to $U$ 's identity $I D_{U}$ and password $p w$ via the value $\alpha$. In the first handshake NCC also authenticated the verifier-table values $\left\{N_{0}, I D_{U}\right\}$ belong to $U$ because these values are verified with the message $M_{1}$ via the value $\alpha$.

\subsection{Data confidentiality and integrity}

The value $r_{1}$ is protected by solving the ECDLP as knowing $P, R$ to find an integer $r_{1}$ such that $R=r_{1} P$ is hard. The session key $S K$ is safeguarded by the secure one-way hash function and mixed with the nonce $N_{1}$ in $\beta=h\left(N_{1}, S K\right)$. The exchange datum can be encrypted with the shared session key $S K$. If an adversary $Z$ eavesdrops and manipulates the messages $M_{1}, M_{2}$ and $M_{3}$, this attack will be quickly detected by checking $\alpha, \beta$ and $\delta$.

\subsection{Perfect session key secrecy}

Supposed an adversary $Z$ captures all the messages $M_{1}, M_{2}$ and $M_{3}$, even extracts the values $\left\{N_{0}, L, v\right\}$ stored in $U$ 's device and steals the values $\left\{N_{0}, I D_{U}\right\}$ in the verifier-table. At first, he may try to compute the value $S=\left(x+h\left(I D_{U}\right)\right)^{-1} P$ as it's important to the system. In one way, $Z$ may apply several users' identity $\left\{I D_{k}\right\}$ to compute values $\left\{P, P_{N}=x P, h\left(I D_{U}\right),\left(h\left(I D_{1}\right),\left(x+h\left(I D_{1}\right)\right)^{-1} P\right), \ldots,\left(h\left(I D_{k}\right),\left(x+h\left(I D_{k}\right)\right)^{-1} P\right)\right\}$

for getting $S$. But, this way is meaningless under the basis of k-CAA1 [17]. Put that problem aside, $Z$ obtains $S$ someway. Then, he tries to compute the session key $S K$ using $S K=k d f\left(N_{0}, I D_{U}, Q^{\prime}\right)$ with $Q^{\prime}=\left(x+h\left(I D_{U}\right)\right)^{-1} R=r_{1}\left(x+h\left(I D_{U}\right)\right)^{-1} P$. But, knowing the pair $(S, R)=\left(\left(x+h\left(I D_{U}\right)\right)^{-1} P, r_{1} P\right)$ to compute the value $Q^{\prime}$ is equivalent to solve the CDHP. On the other hand, he may apply $S K^{\prime}=k d f\left(N_{0}, I D_{U}, Q\right)$ with $Q=r_{1}(y \circ L)=r_{1} S$. But, $r_{1}$ is a secret value. In addition, if the current session key is compromised, $Z$ cannot obtain the forward/backward session keys as each session key is independent depending on the random number $r_{1}$ and the nonce $N_{0}$.

\subsection{User's privacy}

$U$ submits $y=h\left(I D_{U}, p w\right)$ instead of the original password to keep his password from NCC. Then a temporary identity $N_{0}$ is issued and is refreshed every session to protect $U$ 's anonymity. Furthermore, there is not any relevant information about $U$ in the messages $M_{1}, M_{2}$ and $M_{3}$, which can be checked whether the captured messages belong to the specific $U$. A vicious adversary may extract the value $v$ from $U$ 's device to launch the off-line password guessing attack, as it's in the form $v=h\left(y, I D_{U}\right)=h\left(h\left(I D_{U}, p w\right), I D_{U}\right)$ with $U$ 's $I D_{U}$ and $p w$. For this issue, we can strengthen security by asking $U$ to choose the high entropy identity on the basis of IBC. Likewise, for the on-line password guessing attack, we set the wrong-input login threshold value $h$ (e.g. $h=3$ ) at each start of the device.

\subsection{No sensitive data maintained by NCC and users}

There are only verifier-tables $\left\{N_{0}, I D_{U}\right\}$ stored in $N C C$ side and datum $\left\{N_{0}, L, v\right\}$ stored in $U$ side. Any of these values are deadly to the proposed scheme. Because the value $N_{0}$ is just a nonce identity of $U$ and is refreshed every session; for $U$ 's identity $I D_{U}$, $U$ holds the master password $p w$; the value $v$ is derived from $v=h\left(y, I D_{U}\right)$ to protect the fatal value $y=h\left(I D_{U}, p w\right)$, and the value $L$ just masks the value $S$ in the form $L=y \circ S$.

\subsection{Low storage, fast computation and update cost}

Comparing the number of parameters stored at NCC side and $U$ side in Chang et al's scheme and ours: the verifier-tables $\left\{N_{0}, I D_{U}, r, s\right\}$ to $\left\{N_{0}, I D_{U}\right\}$, the devices $\left\{N_{0}, l\right\}$ to $\left\{N_{0}, N_{1}, L, v\right\}$, there are equal parameters in all. However, 160-bit ECC and 1024-bit discrete logarithm problem (DLP) have the same security level [15]. Hence, in the same security level, our parameter storage cost is less than $84 \%$ of in Chang et al's. The operations comparison is shown in Table1. The performance of operations is ranked from high to low under the rules in [14]. The elliptic curve scalar point multiplication (PM) is much less than the modular exponentiation (ME) [15]. From Table 1 and considering the computational bits, the 
whole computation cost of our scheme is much less than Chang's and Li's schemes, even additional the password change phase which is rarely executed in practice. From the storage requirements and computation cost at NCC side, the update of our system is still faster than Chang's scheme.

\subsection{A new denial of service (DoS) attack}

This attack highlights the weakness of the LSC system by jamming a message in application layer [8]. As outlined in Fig.1, we address the problem particularly, which we called 'De-synchronization Challenge'. Generally, $U$ uses his temporary identity $N_{0}$ to login the system and goes to the exchange data phase. However, if an attacker jams the communicated messages, $U$ can still use $N_{0}$ to login. Because the scheme applies three-round handshakes identification technique to ensure the synchronization of $U$ 's temporary identity $N_{0}$ between the $N C C$ and $U . N C C$ updates $N_{0}$ only if it confirms $U$ received the next temporary identity $N_{1}$ in the third handshake. Therefore, as long as the interference is not continuous, $U$ will login the system at last.

The security comparison among related schemes is summarized in Table 2. Distinctly, our protocol provides more security attributes.

Table 1 Operations comparison among related schemes

\begin{tabular}{|c|c|c|c|}
\hline & \multirow[t]{2}{*}{ Registration Phase } & \multicolumn{2}{|c|}{ Authentication Phase } \\
\hline & & $\mathrm{U}$ & $\mathrm{NCC}$ \\
\hline Chang et al. [9] & $1 \mathrm{ME}+2 \mathrm{MO}+1 \mathrm{I}+2 \mathrm{M}+1 \mathrm{~A}+2 \mathrm{H}+1 \mathrm{X}$ & $3 \mathrm{H}+5 \mathrm{X}$ & $1 \mathrm{MO}+3 \mathrm{M}+1 \mathrm{~S}+8 \mathrm{H}+1 \mathrm{X}$ \\
\hline Li et al. $[10]$ & $2 \mathrm{ME}+2 \mathrm{MO}+1 \mathrm{~A}+2 \mathrm{H}$ & $4 \mathrm{ME}+4 \mathrm{MO}+1 \mathrm{I}+5 \mathrm{H}$ & $3 \mathrm{ME}+4 \mathrm{H}+3 \mathrm{MO}$ \\
\hline Proposed & $1 \mathrm{PM}+1 \mathrm{I}+1 \mathrm{~A}+3 \mathrm{H}+1 \mathrm{X}$ & $2 \mathrm{PM}+5 \mathrm{H}+1 \mathrm{X}$ & $1 \mathrm{PM}+1 \mathrm{I}+1 \mathrm{~A}+5 \mathrm{H}$ \\
\hline
\end{tabular}

Table 2 Security comparison among related schemes

\begin{tabular}{lcccc}
\hline Security attributes & Chen et al.[7] & Lasc et al.[8] & Chang et al.[9] & Proposed \\
\hline Impersonation attack & NO & NO & NO & YES \\
Modification attack & NO & YES & NO & YES \\
Stolen-verifier & NO & NO & NO & YES \\
A lost smartcard attack & NO & NO & NO & YES \\
Session key problem & NA & NA & NO & YES \\
On-line and off-line guessing & NA & NA & NA & YES \\
A new denial of service attack & NO & YES & NO & YES \\
Reply attack & YES & YES & YES & YES \\
\hline YES: prevents the attack; NO: does not prevent; NA: not address the attack.
\end{tabular}

\section{CONCLUSIONS}

In this paper, we introduced an enhanced authentication protocol using ECC and IBC with three-round challenge-response identification system. As a result, combining with the superiority of the LSC system, the scheme can be used in multifarious applications, such as Big Data environment.

\section{REFERENCES}

[1] Yiltas D.H, Zaim A. Evaluation of call blocking probabilities in LSC satellite networks. Int J Satell Commun Netw 2009; 27(2):103-115.

[2] Rendon-Morales E, Mata-Díaz J. Performance evaluation of transmission control protocol variants over a satellite multimedia system with QoS. International Journal of Communication Systems 2013. DOI: 10.1002/dac.2333

[3] Shahriar A, Atiquzzaman M, Ivancic W. Network mobility in satellite networks: architecture and the protocol. International Journal of Communication Systems 2014; 26(2):177-197.

[4] Cruickshank H.S. A security system for satellite networks. IEEE Satellite System Mobile Communication Navigation. UK 1996; 187-190.

[5] Hwang M.S, Yang C.C. An authentication scheme for mobile satellite communication systems. ACM SIGOPS Operating Systems Review October 2003; 37(4):42-47.

[6] Chang Y.F, Chang C.C. An efficient authentication protocol for mobile satellite communication systems. ACM SIGOPS Operating Systems. 2005; 39(1):70-84.

[7] Chen T.H, Lee W.B. A self-verification authentication mechanism for mobile satellite communication systems. Computers \& Electrical Engineering 2009; 35(1):41-48.

[8] Lasc L, Dojen R, Coffey T. Countering jamming attacks against an authentication and key agreement protocol for mobile satellite communications. Computers \& Electrical Engineering. 2011; 37(2):160-168.

[9] Chang CC, Cheng TF. An authentication and key agreement protocol for satellite communications. International Journal of Communication Systems 2012. DOI: $10.1002 /$ dac. 2448

[10] Li X, Niu J.W. An enhanced smart card based remote user password authentication scheme. Journal of Network and Computer Applications. 2013 (36) 1365-1371

[11] Chen C.L, Cheng K.W. An improvement on the selfverification authentication mechanism for a mobile satellite communication system. Applied Mathematics \& Information Sciences.2013; 7(1):365-374.

[12] Zhang Y.Y, Chen J.H, H B.J. Security analysis of an authentication and key agreement protocol for satellite communications. International Journal of Communication Systems 2013. DOI: 10.1002/dac.2612

[13] Zheng G, Ma H.T. Design and logical analysis on the access authentication scheme for satellite communication network. ET Information Security 2012; 6(1):6-13.

[14] Menezes AJ, Oorschot PC, Vanstone SA. Handbook of Applied Cryptography. CRC Press, USA, October 1996.

[15] H. D, M. A. Guide to elliptic curve cryptography. New York, USA: LNCS, Springer-Verlag 2004.

[16] He D.B, Chen J.H, Hu J. An ID-based client authentication with key agreement protocol for mobile client-server environment on ECC with provable security. Information Fusion, 13(2012), 223-230. 